\title{
Medium factors affecting extracellular protease activity by Bacillus sp. HTS 102-A novel wild strain isolated from Portuguese merino wool
}

\author{
Ana Catarina Queiroga ${ }^{1,2}$, Manuela Estevez Pintado ${ }^{1}$, Francisco Xavier Malcata ${ }^{3,4^{*}}$ \\ ${ }^{1} \mathrm{CBQF}$, Porto, Portugal \\ ${ }^{2}$ Instituto Superior de Agronomia, Lisboa, Portugal \\ ${ }^{3}$ Department of Chemical Engineering of University of Porto, Porto, Portugal; *Corresponding Author: fmalcata@,fe.up.pt \\ ${ }^{4}$ ITQB, Oeiras, Portugal
}

Received 21 April 2013; revised 22 May 2013; accepted 31 May 2013

Copyright (C) 2013 Ana Catarina Queiroga et al. This is an open access article distributed under the Creative Commons Attribution License, which permits unrestricted use, distribution, and reproduction in any medium, provided the original work is properly cited.

\begin{abstract}
The synthesis of an extracellular protease by Bacillus sp. HTS102-a wild strain recently isolated from the wool of Portuguese Merino ewes, was optimized. This protease is thermostable and particularly resistant to harsh environmental conditions-and appears to bear a unique ability to hydrolyze keratin-rich solid materials. Following a preliminary screening for the most relevant medium factors involved in processing, a fractional factorial design $\left(2 \mathrm{vI}^{6-1}\right)$ was applied to ascertain the effects of six relevant parametersviz. yeast extract concentration, peptone level, inoculum size, stirring rate, temperature and $\mathrm{pH}$. The concentrations of yeast extract and peptone, as well as the incubation temperature and $\mathrm{pH}$ were found to play significant roles; and the 2-factor interaction between yeast extract level and $\mathrm{pH}$ was also significant. A 2.2-fold increase in the overall level of protease synthesis was eventually attained, with the improved medium relative to the basal medium-which is noteworthy when compared with competing proteases and previous optimization efforts.
\end{abstract}

Keywords: Fractional Factorial Design;

Thermotolerant Enzyme; Proteolytic Activity;

Bacillus sp.; Ewe's Wool

\section{INTRODUCTION}

Use of enzymes for industrial processing has received considerable attention in recent years [1], owing mainly to environmental concerns; and proteases on their own already account for ca. $40 \%$ of the total enzyme sales worldwide [2]. Proteases are indeed essential for cell growth and differentiation, so they are ubiquitously found in living organisms [3] — with Alcaligenes faecalis, Pseudomonas fluorescens, Pseudomonas aeruginosa, Bacillus cereus, Bacillus licheniformis, Bacillus lentus, Clostridium sp. and Aeromonas hydrophilica accounting for some of the highest rates of synthesis observed to date $[2,4]$. Extracellular proteases are particularly convenient from a production standpoint, and large productivities have accordingly been observed for several species of the Bacillus genus [3].

As a consequence of the large array of commercial proteases available at present, an ever-growing portfolio of uses has also been witnessed, yet higher and higher demand has driven comprehensive screening efforts for new strains of bacteria that can produce proteases with better features and/or be susceptible to development of lower cost/higher throughput industrial processes $[5,6]$. For instance, an organic solvent-stable protease from $\mathrm{Ba}$ cillus sphaericus (Liu et al., 2010) and a milk-clotting proease from Bacillus subtilis (Fang et al., 2009) were described; however, our native strain appears to be quite distinct, so any better achievements claimed elsewhere (among a rather voluminous literature) and pertaining to different species are not necessarily paralleled by our unique strain.

It has for long been recognized that one major obstacle to successful biotechnological applications in the industry at large is the overall costs of enzyme production and downstream purification [2]; $30 \%-40 \%$ of the final cost of enzyme arises indeed from the culture medium used for fermentation of its source strain [7], so optimization of fermentation will assume a great relevance if economically feasible processes are sought.

A number of comprehensive efforts have been devel- 
oped by biochemists and biochemical engineers to increase protease yields [2], yet no ideal or all-purpose medium for maximum microbial protease production has been formulated to date. Each microorganism has in fact its own specific growth and enzyme synthesis/secretion constraints, so unique physicochemical and nutritional requirements are found [8]. Therefore, the qualitative and quantitative profiles of the culture medium, further to other operating factors, are to be optimized for each specific microorganism and desired metabolite. For instance, extracellular protease production is strongly influenced by such medium features as $\mathrm{C} / \mathrm{N}$ ratio, presence/absence of readily metabolizable sugars and nitrogen sources, and availability of specific metal ions; however, such processing conditions as rate of aeration, inoculum density, $\mathrm{pH}$, temperature and incubation time also play a crucial role [2].

Optimization of processing parameters (encompassing also medium components) by classical methods that involve change of "one-variable-at-a-time" is extremely time-consuming, and thus expensive when a large number of variables is to be considered [9]. Despite ignoring the interactions among parameters, this is still the most frequently used strategy in bioprocess engineering to obtain high yields of enzyme in microbial systems [10]especially at earlier stages of process optimization, when little is known about the factors that actually affect enzyme synthesis yield and rate. As a result, preliminary studies ignoring mutual processing interactions are acceptable just to find whether or not one (or several) factor(s) under scrutiny affect protease production to a significant extent; the most reasonable ranges for further optimization, encompassing interactions between those factors [11], are thus in order.

To overcome the aforementioned inability of the "oneat-a-time" approach to pinpoint interactions among the processing parameters, factorial designs have been proposed [10]. These designs are in fact frequently used in screening for key factors that affect the response when more than 3 factors are to be tested; they usually assume a suitable fractional form to avoid the need for an excessively large number of experiments [11].

The major goal of this research effort was thus to find the best combination of processing factors aimed at improving protease production by the wool-associated $\mathrm{Ba}$ cillus sp. strain HTS 102. This is a novel strain recently isolated from a Portuguese autochthonous sheep breed that excretes a very strong and stable protease exhibiting keratinolytic features. After conducting several comparative "one-factor-at-a-time" studies, a $2{ }_{\mathrm{VI}}^{6-1}$ fractional factorial design was applied to better elucidate the main effects and two-factor interactions associated with the most promising processing parameters - and eventually to increase the protease productivity of that microorgan- ism to an extent sufficient to backup eventual interest from industry.

\section{MATERIALS AND METHODS}

\subsection{Preliminary Screening via "One-Factor-at-a-Time" Approach}

\subsubsection{Reference Culture Medium}

The Nutrient Broth (NB) medium was used as control; although this may not be the least expensive medium available for industrial scale up, its stability in terms of composition was found to be determinant of technological reproducibility - and thus led our selection over putative media obtained from cheaper sources. It was prepared according to the recipe provided by the manufacturer (Lab M, UK), but using ingredients added separately, viz.: $2.0 \mathrm{~g} \cdot \mathrm{L}^{-1}$ yeast extract (Biokar Diagnostics, France), $1.0 \mathrm{~g} \cdot \mathrm{L}^{-1}$ beef extract (Merck, Germany), 5.0 $\mathrm{g} \cdot \mathrm{L}^{-1}$ peptone (Sigma-Aldrich, USA) and $5.0 \mathrm{~g} \cdot \mathrm{L}^{-1} \mathrm{NaCl}$ (Carlo Erba, Italy).

\subsubsection{Carbon/Nitrogen Source Selection}

The beef and yeast extracts, referred to in the previous section, were separately replaced with alternative (and less expensive) carbon and nitrogen sources, respectively, at equivalent concentrations. Carbon sources tested were starch, glycerol, lactose, sucrose, glucose and fructose. Nitrogen sources considered were peptone, tryptone, ammonium chloride and ammonium sulphate.

\subsubsection{Significant Physicochemical Parameter Determination}

A total of 10 physicochemical factors, which had been reported previously to influence protease production in a number of bacteria, were tested in attempts to find those critical for protease production by Bacillus sp. HTS 102 . Such factors were metal ion concentration-10 $\mathrm{mM}$ $\mathrm{FeSO}_{4}$ and $0.1 \mathrm{~g} \cdot \mathrm{L}^{-1} \mathrm{MgSO}_{4}$, inoculum density, $\mathrm{pH}$ value, incubation temperature, stirring rate, and presence of $0.8 \%$ (w/v) $\mathrm{Na}_{2} \mathrm{CO}_{3}, 10 \mathrm{mM} \mathrm{CaCl}, 0.02 \%$ (v/v) Triton $\mathrm{X}-100$ and $1.0 \mathrm{~g} \cdot \mathrm{L}^{-1} \mathrm{~K}_{2} \mathrm{HPO}_{4}$.

\subsubsection{Nutrient Broth Constituent Effects}

Several medium formulations were prepared by removing "one-constituent-at-a-time", so as to ascertain the effect of each constituent of NB upon protease production by the target bacterium. The media assayed for are tabulated in Table $\mathbf{1 .}$

\subsection{Optimization via $2 \mathrm{vl}^{6-1}$ Fractional Factorial Design Approach}

After selection of a total of six key factors for protease production by Bacillus sp. HTS102, the levels of yeast 
extract and peptone, inoculum density, stirring rate, $\mathrm{pH}$ value and incubation temperature were optimized via a $2{ }_{\mathrm{VI}}^{6-1}$ fractional factorial, unreplicated design. The range of values for each factor was chosen based on information available in the literature, coupled with experience meanwhile gained during the "one-factor-at-a-time" comparative studies. The ranges of values used within such a fractional factorial design are depicted in Table 2.

\subsection{Fermentation Conditions}

Erlenmeyer flasks $(250 \mathrm{~mL})$ containing $25 \mathrm{~mL}$ of growth medium were inoculated with $1 \%(\mathrm{v} / \mathrm{v})$ fresh inoculum (0.6 O.D.600nm). The extracellular protease activity was quantitated after a $36 \mathrm{~h}$-incubation period at $37^{\circ} \mathrm{C}$, unless stated otherwise. The microorganism used in this study was Bacillus sp. HTS 102 (GenBank accession number HQ698269), isolated from Portuguese Merino wool [12] - and formally deposited in a publicly accessible culture collection (LMG 26323, from BCCM/ LMG Bacterial Culture Collection, Ghent, Belgium).

Table 1. Characterization of medium formulations, based on Nutrient Broth (NB), tested to enhance protease productivity.

\begin{tabular}{|c|c|c|c|c|c|}
\hline \multirow{2}{*}{$\begin{array}{l}\text { Formulation } \\
\text { code }\end{array}$} & \multicolumn{4}{|c|}{ Nutrients (g/L) } & \multirow[t]{2}{*}{ Description } \\
\hline & $\begin{array}{l}\text { Yeast } \\
\text { extract }\end{array}$ & $\begin{array}{l}\text { Beef } \\
\text { extract }\end{array}$ & Peptone & $\mathrm{NaCl}$ & \\
\hline NB-C & 2 & 1 & 5 & 5 & NB complete \\
\hline NB-Y & 0 & 1 & 5 & 5 & $\begin{array}{c}\text { NB without yeast } \\
\text { extract }\end{array}$ \\
\hline NB-B & 2 & 0 & 5 & 5 & $\begin{array}{c}\text { NB without beef } \\
\text { extract }\end{array}$ \\
\hline NB-P & 2 & 1 & 0 & 5 & NB without peptone \\
\hline NB-N & 2 & 1 & 5 & 0 & $\mathrm{NB}$ without $\mathrm{NaCl}$ \\
\hline NB-YN & 0 & 1 & 5 & 0 & $\begin{array}{l}\mathrm{NB} \text { without yeast } \\
\text { extract and } \mathrm{NaCl}\end{array}$ \\
\hline
\end{tabular}

Table 2. Definition of processing parameters, in original and coded format, within a $2{ }_{\mathrm{VI}}^{6-1}$ experimental design, tested to enhance protease productivity.

\begin{tabular}{ccccc}
\hline & & & \multicolumn{2}{c}{$\begin{array}{c}\text { Actual values } \\
\text { Corresponding to } \\
\text { parameter } \\
\text { coded levels }\end{array}$} \\
\cline { 3 - 5 } & Alias & Description & -1 & +1 \\
\hline $\mathrm{X}_{1}$ & 1 & Concentration of yeast $(\mathrm{g} / \mathrm{L})$ & 5.0 & 10.0 \\
$\mathrm{X}_{2}$ & 2 & Concentration of peptone $(\mathrm{g} / \mathrm{L})$ & 2.0 & 4.0 \\
$\mathrm{X}_{3}$ & 3 & Level of inoculum $(\%, \mathrm{v} / \mathrm{v})$ & 1.0 & 3.0 \\
$\mathrm{X}_{4}$ & 4 & Stirring rate $(\mathrm{rpm})$ & 0 & 100 \\
$\mathrm{X}_{5}$ & 5 & Temperature $\left({ }^{\circ} \mathrm{C}\right)$ & 34 & 40 \\
$\mathrm{X}_{6}$ & 12345 & $\mathrm{pH}$ & 6.0 & 8.0 \\
\hline
\end{tabular}

\subsection{Protease Activity Assay}

The cell-free supernatants, sterilized by filtration through a $0.45 \mu \mathrm{m}$ filter, were assayed for protease activity using a colorimetric determination of casein breakdown extent at $660 \mathrm{~nm}$, resorting to Folin-Ciocalteu's reagent, according to supplier's instructions (Sigma-Aldrich). One unit (U) of proteolytic activity was defined as the amount of enzyme able to hydrolyse casein so as to produce an absorbance variation per min equal to that produced by $1.0 \mu \mathrm{mol}$ of tyrosine, at $\mathrm{pH} 7.5$ at $37^{\circ} \mathrm{C}$.

\subsection{Protein Content Assay}

Throughout the $36 \mathrm{~h}$-incubation period, aliquots were withdrawn for total protein assay via absorbance at 562 $\mathrm{nm}$ using the $\mathrm{BCA}^{\mathrm{TM}}$ Protein Assay Kit (Pierce, USA), again according to supplier's instructions.

\subsection{Data Statistical Analysis}

Unless otherwise stated, all data presented here result from two independent experiments, each run in duplicate.

The means of each treatment following the "one-factor-at-a-time" approach were compared using one-way analysis of variance (ANOVA), at a significance level of $5 \%$; a supplementary multiple comparison test (TukeyHSD) was employed to pinpoint which pairs of means were statistically different from each other at that significance level.

The statistical software package Design-Expert ${ }^{\circledR}$, v. 8.0.3 (Stat-Ease, Minneapolis MN, USA) was used to set up and analyse the results of the fractional factorial designs. The statistical significance of the underlying linear model equation and of its terms was assessed via Fischer's F-tests. The quality-of-fit of said polynomial model equation was expressed by a coefficient of determination $\left(\mathrm{R}^{2}\right)$ and an adjusted $\mathrm{R}^{2}$. The fitted polynomial equation was represented as contour plots for the two-factor interactions only, to better illustrate the underlying relationships between response and level tested of each processing parameter.

\section{RESULTS}

\subsection{Carbon/Nitrogen Source Selection}

Of the several carbon sources tested, those of the original NB medium formulation yielded by far the highest protease activity (Figure 1(a)). As will be discussed later, production of protease is almost fully repressed in the presence of glucose, so carbon source was not included as optimization factor thereafter. With regard to the nitrogen sources, all tested inorganic and organic compounds appeared to hamper enzyme production relative to that observed in the control using plain 
(a)

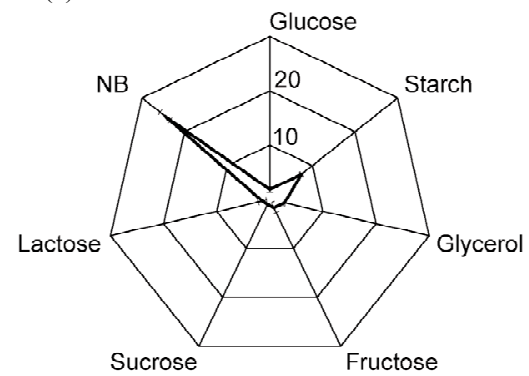

(d) (b)

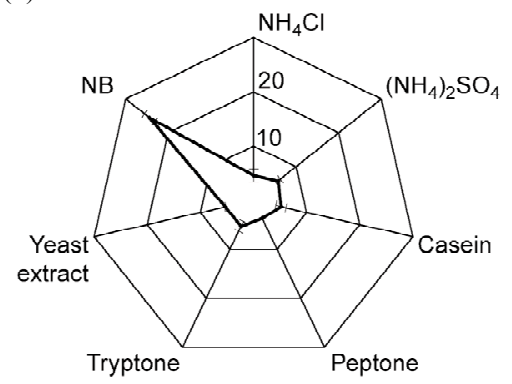

(f)

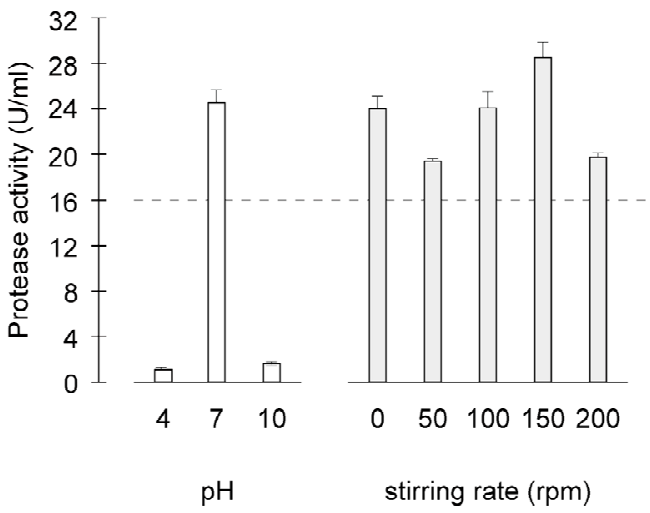

(c)

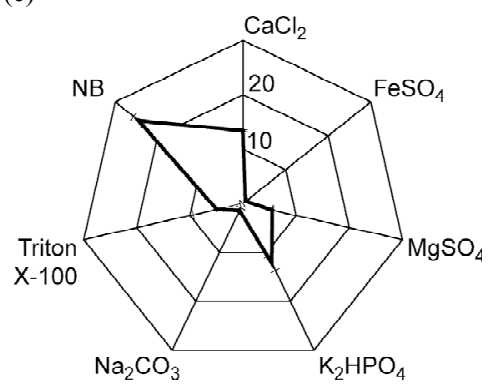

(h)

Figure 1. Results of trials following a preliminary "one-factor-at-a-time" approach, aimed at screening for relevant overall factors upon protease activity: (a) Effects of carbon sources $\left(g \cdot \mathrm{L}^{-1}\right)$; (b) Effects of nitrogen sources $\left(\mathrm{g} \cdot \mathrm{L}^{-1}\right)$; and (c) Effects of salts and surfactants $\left(\mathrm{g} \cdot \mathrm{L}^{-1}\right)$, using a star layout; and (d) Effect of $\mathrm{pH}$; (e) Effect of stirring; (f) Effect of temperature; (g) Effect of inoculum level; and (h) Effect of NB medium constituents.

NB (Figure 1(b)). When yeast extract and peptone were replaced by plain peptone, the production of protease was remarkably reduced, thus suggesting that yeast extract and peptone may act synergistically.

\subsection{Significant Physicochemical Parameter Determination}

To ascertain the effects of distinct ingredients (including metals) upon protease activity, the reference medium (NB) was enriched therewith on a "one-by-one" basis. All components tested led to lower protease production than the reference medium (Figure 1(c)).

The effect of initial $\mathrm{pH}$ upon protease production was sought at $\mathrm{pH}$ values 4, 7 and 10 (Figure 1(d)). These results showed that Bacillus sp. HTS 102 was able to grow and release protease in a broad $\mathrm{pH}$ range.

The stirring rate affected protease production according to a "U-type" pattern (Figure 1(e)). In all four assays carried out (i.e. two independent experiments, each run in duplicate), horizontal orbital shaking of the culture at $50 \mathrm{rpm}$ yielded a lower protease activity than any other rate tested. Surprisingly, no stirring at all produced essentially similar effects as stirring at rates above $50 \mathrm{rpm}$.

The maximum protease activity was attained at $37^{\circ} \mathrm{C}$
(Figure 1(f)), whereas dramatically lower levels were obtained at 30 and $55^{\circ} \mathrm{C}$, i.e. 2.3 and $2.5 \%$, respectively, of the activity observed at $37^{\circ} \mathrm{C}$.

The inoculum level affected growth to a considerable degree, but not protease synthesis (Figure 1(g)). The highest protease production was achieved at an inoculum size of $4 \%$, even though the difference between $2 \%$ - and $4 \%$-inoculum levels was not statistically significant ( $\mathrm{P}>$ $0.05)$.

\subsection{Nutrient Broth Constituent Effects}

The nutrient broth constituent that affected the most (P $<0.05)$ the production of protease was beef extract (Figure 1(h)). Nevertheless, every other constituent of the base NB medium played a role upon production of enzyme, because its exclusion along a "one-at-a-time" approach greatly reduced enzyme synthesis. Furthermore, when yeast extract and $\mathrm{NaCl}$ were simultaneously excluded from the medium, a further decrease in protease production was observed - thus indicating that $\mathrm{NaCl}$, despite not being a source of nitrogen or carbon, probably plays a role in assuring the appropriate ionic strength for protease synthesis.

The design matrix of the $2_{\mathrm{IV}}{ }^{4-1}$ experiments, and the 
corresponding protease activity measurements and predictions by the underlying polynomial are depicted in Figure 2. The data obtained from the $2_{\mathrm{IV}}{ }^{4-1}$ experiments were also subjected to analysis of variance (ANOVA). A half-normal probability plot of the estimates of the effects (Figure 3(a)) permitted selection of the significant terms using Fischer's F-test as tool [13]. The ANOVA results indicated that the interactions between beef extract and yeast extract were significant (Figure 3(b)), whereas the linear effects of beef extract and yeast extract were unexpectedly not significant; however, these

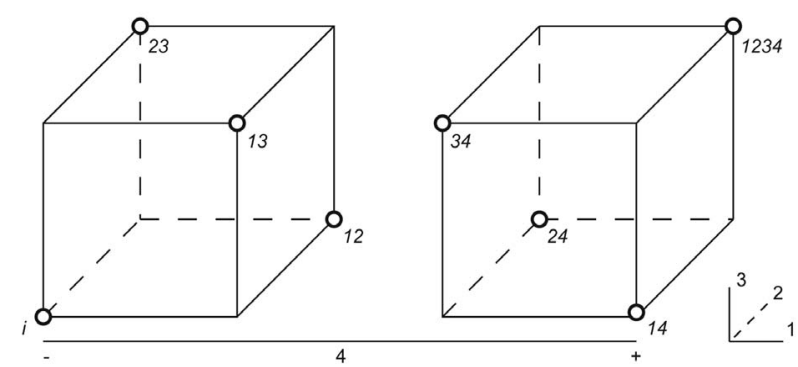

(a)

\begin{tabular}{ccccc}
\hline \multirow{2}{*}{$\begin{array}{c}\text { Coded } \\
\text { parameters }\end{array}$} & Alias & Description & \multicolumn{2}{c}{$\begin{array}{c}\text { Actual values corresponding } \\
\text { to coded values }\end{array}$} \\
\cline { 4 - 5 } & & & -1 & +1 \\
\hline $\mathrm{X}_{1}$ & 1 & Beef extract $(\mathrm{g} / \mathrm{L})$ & 0.5 & 1.5 \\
$\mathrm{X}_{2}$ & 2 & Yeast extract $(\mathrm{g} / \mathrm{L})$ & 2.5 & 7.5 \\
$\mathrm{X}_{3}$ & 3 & $\mathrm{NaCl}(\mathrm{g} / \mathrm{L})$ & 1.0 & 3.0 \\
$\mathrm{X}_{4}$ & 123 & Peptone $(\mathrm{g} / \mathrm{L})$ & 1.0 & 3.0 \\
\hline
\end{tabular}

(b)

\begin{tabular}{|c|c|c|c|c|c|c|c|c|}
\hline \multirow{2}{*}{ Exp } & \multirow{2}{*}{$\begin{array}{l}\text { Run } \\
\text { order }\end{array}$} & \multicolumn{4}{|c|}{ Coded parameter } & \multirow{2}{*}{ Alias } & \multicolumn{2}{|c|}{$\begin{array}{l}\text { Protease activity } \\
(\mathrm{U} / \mathrm{mL})\end{array}$} \\
\hline & & $\mathrm{X}_{1}$ & $\mathrm{X}_{2}$ & $\mathrm{X}_{3}$ & $\mathrm{X}_{4}$ & & Observed & Predicted \\
\hline 1 & 4 & -1 & -1 & -1 & -1 & $\mathrm{i}$ & 20.50 & 20.27 \\
\hline 2 & 3 & +1 & -1 & -1 & +1 & 14 & 41.27 & 40.57 \\
\hline 3 & 8 & -1 & +1 & -1 & +1 & 24 & 43.42 & 40.78 \\
\hline 4 & 1 & +1 & +1 & -1 & -1 & 12 & 22.62 & 24.43 \\
\hline 5 & 6 & -1 & -1 & +1 & +1 & 34 & 20.05 & 20.27 \\
\hline 6 & 5 & +1 & -1 & +1 & -1 & 13 & 39.88 & 40.57 \\
\hline 7 & 2 & -1 & +1 & +1 & -1 & 23 & 38.13 & 40.78 \\
\hline 8 & 7 & +1 & +1 & +1 & +1 & 1234 & 26.25 & 24.43 \\
\hline
\end{tabular}

(c)

Figure 2. Results of trials following a $2_{\mathrm{IV}}{ }^{4-1}$ fractional factorial design, aimed at elucidating the effects of key medium constituents upon protease activity: (a) Geometric representation of design; (b) Definition of processing parameters and their testing range; and (c) Design matrix with observed and associated predicted values. terms could not be excluded from the model, because of the need to satisfy hierarchical conditions. The high overall $F$-value found (42.38) indicates that the postulated model provides a good fit-with a mere $0.17 \%$ chance that such a large value occurs due to pure noise (Figure 3(b)).

Our experimental data were thus fitted to by the following two-factor interaction model equation:

$$
\mathrm{Y}=\beta_{0}+\sum_{i=1}^{4} \beta_{i} X_{i}+\sum_{i=1}^{3} \sum_{j=i+1}^{4} \beta_{i j} X_{i} X_{j}
$$

where $Y$ is the predicted response, $\beta_{0}$ is the intercept term, $\beta_{i}$ 's are the linear effects, and $\beta_{\mathrm{ij}}$ 's are the interaction effects, with $X_{i}$ and $X_{j}$ being the processing variables.

In order to determine the levels of each variable that led to maximum protease production, contour plots were constructed by representing the protease activity versus any two independent variables, while maintaining the

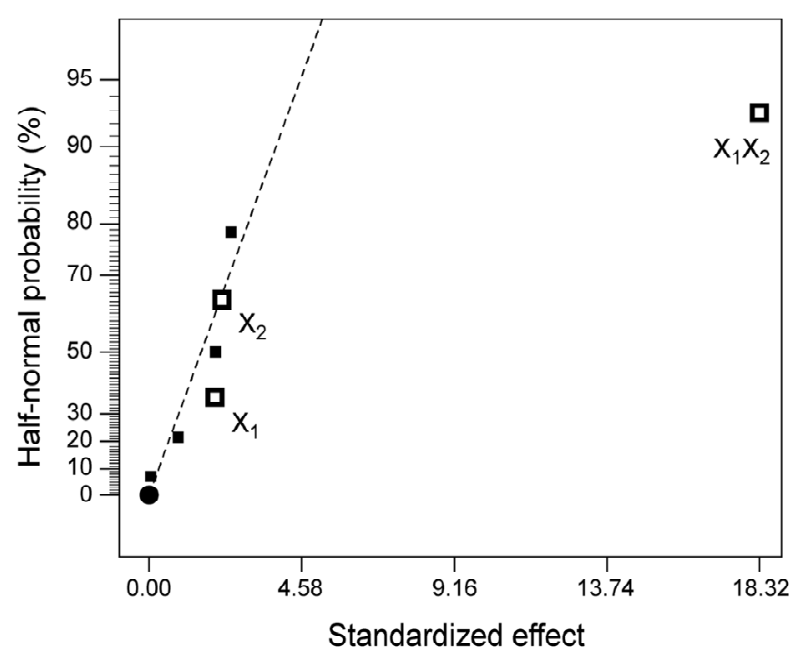

(a)

\begin{tabular}{cccccc}
\hline Source & SS & dF & MS & F & $\mathrm{p}$ \\
\hline Model & 688.76 & 3 & 229.59 & 42.38 & 0.0017 \\
$\mathrm{X}_{1}$ & 7.83 & 1 & 7.83 & 1.45 & 0.2955 \\
$\mathrm{X}_{2}$ & 9.52 & 1 & 9.52 & 1.76 & 0.2556 \\
$\mathrm{X}_{1} \mathrm{X}_{2}$ & 671.41 & 1 & 671.41 & 123.93 & 0.0004 \\
Residual & 21.67 & 4 & 5.42 & & \\
Total & 710.44 & 7 & & & \\
\hline
\end{tabular}

SS: Sum of Squares; dF: degrees of Freedom; MS: Mean Square; F: F ratio; p: probability value.

Figure 3. Results of analysis of variance encompassing the parameters with lowest individual linear effects, but with the highest interaction effects upon protease activity: (a) Half-normal probability plot of effects $\left(\mathrm{R}^{2}=0.9695\right.$; adj $\mathrm{R}^{2}=0.9466$; pred $\mathrm{R}^{2}=0.8780$; adeq precision $\left.=12.458\right)$; and $(\mathrm{b})$ Associated probabilities. 
remaining variables at their optimum levels. As shown in Figure 4, an increase in protease activity was observed when the beef extract concentration increased and the yeast extract concentration decreased (should all other linear factors remain constant). A similar trend held when the concentration of beef extract decreased and the concentration of yeast extract increased, so these variables do indeed interact with each other.

\subsection{Optimization via $2 \mathrm{vl}^{6-1}$ Fractional Factorial Design}

The $2{ }_{\mathrm{VI}}^{6-1}$ fractional factorial experimental design was aimed at determining the ingredients that most strongly affected the rate of protease synthesis; there were accordingly six factors. Each of said factors was examined at a higher $(+1)$ and a lower $(-1)$ level; the design variables and their levels are provided in detail in Table 2.

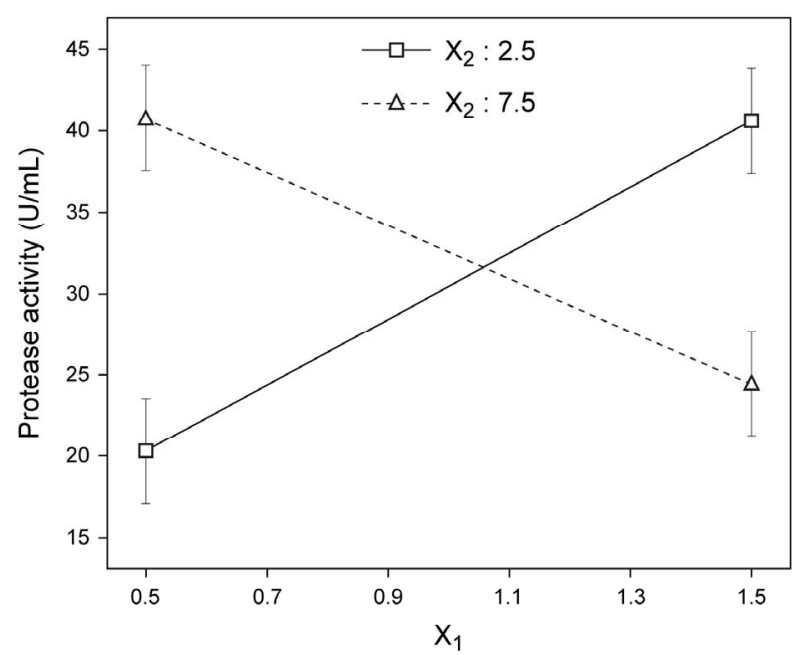

(a)

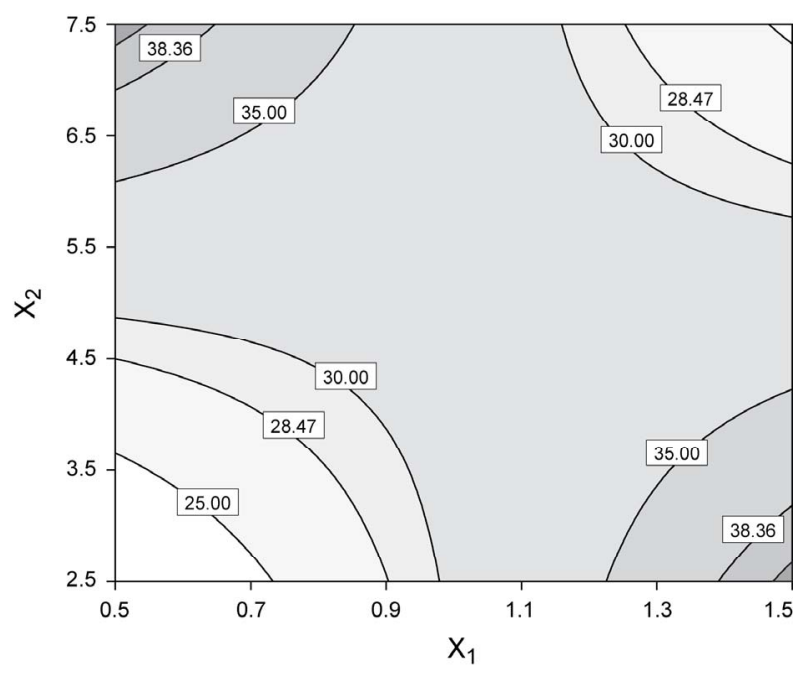

(b)

Figure 4. Best fit model plots: (a) Interaction plot; and (b) Contour plot associated with the significant $\mathrm{X}_{1} \mathrm{X}_{2}$ interaction.
One half fraction of the full factorial design was adopted, thus encompassing the 32 treatments shown in Figure 5.

The $2{ }_{\mathrm{VI}}^{6-1}$ fractional factorial design is described in Table 2, and the corresponding results are illustrated in Figures 5 and 6. The protease activity measured varied widely from 7.40 and $54.64 \mathrm{U} \cdot \mathrm{mL}^{-1}$, among the various combinations of processing factors studied. From the results of the linear regression analysis based on the $2_{\mathrm{VI}}{ }^{6-1}$ fractional factorial design, one was led to the following simplified form:

$$
\begin{aligned}
Y= & -4.69+1.20 X_{1}-0.36 X_{2}+0.18 X_{5} \\
& +0.80 X_{6}-0.19 X_{1} X_{6}
\end{aligned}
$$

The associated regression and determination coefficients for the above model are given in Figure 6(b); they and unfold a high significance, owing to the associated large $F$-value and $\mathrm{R}^{2}$ value close to unity. Furthermore, the model held an "adequate precision" value of 22.038 .

Using the whole information produced in this research effort, the maximum protease activity was reached under the following fermentation conditions: $10 \mathrm{~g} \cdot \mathrm{L}^{-1}$ yeast extract, $2 \mathrm{~g} \cdot \mathrm{L}^{-1}$ peptone, $3 \%(\mathrm{v} / \mathrm{v})$ inoculum, $100 \mathrm{rpm}$ stirring rate, $40^{\circ} \mathrm{C}$ and $\mathrm{pH}$ 6. Furthermore, a less expensive fermentation medium could be attained by reducing the inoculum size to just $1 \%(\mathrm{v} / \mathrm{v})$; and less expensive operating conditions were feasible by eliminating stirring, since that would not significantly compromise our final goal.

\section{DISCUSSION}

The first stage in developing industrial bioprocesses is to isolate a strain able to produce a target metabolite (or a related one) to sufficiently high yields [14]. This approach entails intensive screening, and thus requires testing of a large number of strains in attempts to pinpoint fast producers, or even alternative useful metabolites. The conventional experimental practice pertaining to extracellular microbial products is to bring about growth on agar plate media, and then assess the microbial capacity of production from the radius of the product zone diffusing away around the source colony; this strategy was described in detail elsewhere [12], when isolating proteaseproducing bacteria intended for novel modifications of wool in the textile industry.

Due to the ever-increasing economic relevance of alkaline proteases, optimization of a large number of fermentation parameters for Bacillus sp. HTS102 was sought - including medium composition and culture conditions, using extracellular protease production as objective function. After having isolated one (or more) strain(s) bearing promising features, one should then proceed to optimization of metabolite synthesis and secretion so as to make its industrial use as appealing as possible. Recall that the optimization protocol followed here encom- 


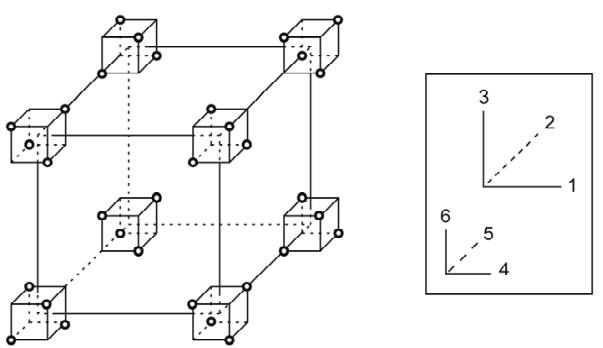

(a)

\begin{tabular}{|c|c|c|c|c|c|c|c|c|c|c|}
\hline \multirow{2}{*}{ Exp } & \multirow{2}{*}{$\begin{array}{l}\text { Run } \\
\text { order }\end{array}$} & \multicolumn{6}{|c|}{ Coded parameter } & \multirow[t]{2}{*}{ Alias } & \multicolumn{2}{|c|}{$\begin{array}{c}\text { Protease activity } \\
(\mathrm{U} / \mathrm{mL})\end{array}$} \\
\hline & & $\mathrm{X}_{1}$ & $\mathrm{X}_{2}$ & $\mathrm{X}_{3}$ & $\mathrm{X}_{4}$ & $\mathrm{X}_{5}$ & $\mathrm{X}_{6}$ & & Observed & Predicted \\
\hline 1 & 10 & -1 & -1 & -1 & -1 & -1 & -1 & $\mathrm{i}$ & 29.80 & 31.81 \\
\hline 2 & 26 & +1 & -1 & -1 & -1 & -1 & +1 & 16 & 15.51 & 13.32 \\
\hline 3 & 12 & -1 & +1 & -1 & -1 & -1 & +1 & 26 & 18.26 & 21.07 \\
\hline 4 & 8 & +1 & +1 & -1 & -1 & -1 & -1 & 12 & 27.22 & 26.63 \\
\hline 5 & 2 & -1 & -1 & +1 & -1 & -1 & +1 & 36 & 25.84 & 28.30 \\
\hline 6 & 1 & +1 & -1 & +1 & -1 & -1 & -1 & 13 & 37.21 & 34.69 \\
\hline 7 & 9 & -1 & +1 & +1 & -1 & -1 & -1 & 23 & 22.76 & 24.11 \\
\hline 8 & 23 & +1 & +1 & +1 & -1 & -1 & +1 & 1236 & 8.02 & 8.53 \\
\hline 9 & 17 & -1 & -1 & -1 & +1 & -1 & +1 & 46 & 26.03 & 28.30 \\
\hline 10 & 20 & +1 & -1 & -1 & +1 & -1 & -1 & 14 & 37.22 & 34.69 \\
\hline 11 & 27 & -1 & +1 & -1 & +1 & -1 & -1 & 24 & 26.68 & 24.11 \\
\hline 12 & 19 & +1 & +1 & -1 & +1 & -1 & +1 & 1246 & 7.4 & 8.53 \\
\hline 13 & 3 & -1 & -1 & +1 & +1 & -1 & -1 & 34 & 36.94 & 31.81 \\
\hline 14 & 14 & +1 & -1 & +1 & +1 & -1 & +1 & 1346 & 16.20 & 13.32 \\
\hline 15 & 18 & -1 & +1 & +1 & +1 & -1 & +1 & 2346 & 18.12 & 21.07 \\
\hline 16 & 16 & +1 & +1 & +1 & +1 & -1 & -1 & 1234 & 25.26 & 26.63 \\
\hline 17 & 21 & -1 & -1 & -1 & -1 & +1 & +1 & 56 & 32.63 & 40.07 \\
\hline 18 & 24 & +1 & -1 & -1 & -1 & +1 & -1 & 15 & 47.30 & 48.30 \\
\hline 19 & 32 & -1 & +1 & -1 & -1 & +1 & -1 & 25 & 37.34 & 35.64 \\
\hline 20 & 6 & +1 & +1 & -1 & -1 & +1 & +1 & 1256 & 12.29 & 15.84 \\
\hline 21 & 31 & -1 & -1 & +1 & -1 & +1 & -1 & 35 & 37.67 & 44.89 \\
\hline 22 & 7 & +1 & -1 & +1 & -1 & +1 & +1 & 1356 & 18.98 & 22.18 \\
\hline 23 & 30 & -1 & +1 & +1 & -1 & +1 & +1 & 2356 & 36.26 & 31.92 \\
\hline 24 & 13 & +1 & +1 & +1 & -1 & +1 & -1 & 1235 & 35.84 & 38.69 \\
\hline 25 & 15 & -1 & -1 & -1 & +1 & +1 & -1 & 45 & 39.97 & 44.89 \\
\hline 26 & 25 & +1 & -1 & -1 & +1 & +1 & +1 & 1456 & 20.33 & 22.18 \\
\hline 27 & 11 & -1 & +1 & -1 & +1 & +1 & +1 & 2456 & 38.14 & 31.92 \\
\hline 28 & 22 & +1 & +1 & -1 & +1 & +1 & -1 & 1245 & 32.71 & 38.69 \\
\hline 29 & 28 & -1 & -1 & +1 & +1 & +1 & +1 & 3456 & 52.88 & 40.70 \\
\hline 30 & 4 & +1 & -1 & +1 & +1 & +1 & -1 & 1345 & 54.64 & 48.30 \\
\hline 31 & 29 & -1 & +1 & +1 & +1 & +1 & -1 & 2345 & 41.09 & 35.64 \\
\hline 32 & 5 & +1 & +1 & +1 & +1 & +1 & +1 & 123456 & 21.32 & 15.84 \\
\hline
\end{tabular}

(b)

Figure 5. Results of trials following a $2 \mathrm{VI}^{6-1}$ design, aimed at elucidating the processing parameters upon protease activity: (a) Geometric representation of design; and (b) Design matrix with observed and associated predicted values.

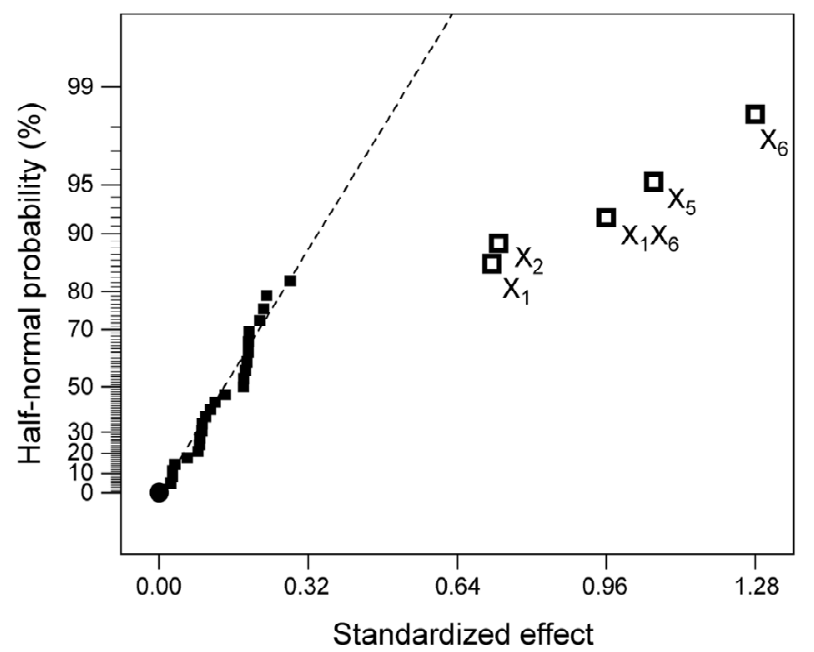

(a)

\begin{tabular}{cccccc}
\hline Source & $\mathrm{SS}$ & $\mathrm{dF}$ & $\mathrm{MS}$ & $\mathrm{F}$ & $\mathrm{p}$ \\
\hline Model & 37.81 & 5 & 7.56 & 42.41 & $<0.0001$ \\
$\mathrm{X}_{1}$ & 4.08 & 1 & 4.08 & 22.88 & $<0.0001$ \\
$\mathrm{X}_{2}$ & 4.25 & 1 & 4.25 & 23.84 & $<0.0001$ \\
$\mathrm{X}_{5}$ & 9.01 & 1 & 9.01 & 50.54 & $<0.0001$ \\
$\mathrm{X}_{6}$ & 13.10 & 1 & 13.10 & 73.45 & $<0.0001$ \\
$\mathrm{X}_{1} \mathrm{X}_{6}$ & 7.37 & 1 & 7.37 & 41.34 & $<0.0001$ \\
Residual & 4.64 & 26 & 0.18 & & \\
Total & 42.44 & 31 & & & \\
\hline
\end{tabular}

SS: Sum of Squares; dF: degrees of Freedom; MS: Mean Square; F: F ratio; p: probability value.

(b)

Figure 6. Results of variance encompassing the parameters with highest individual linear effects upon protease activity: (a) Half-normal probability plot of effects $\left(\mathrm{R}^{2}=0.8908\right.$; adj $\mathrm{R}^{2}=$ 0.8698 ; pred $\mathrm{R}^{2}=0.8345$; adeq precision $\left.=22.038\right)$; and $(\mathrm{b})$ Associated probabilities.

passed two sequential approaches: first, an "one-factorat-a-time" set of experiments performed with a total of 24 factors, so as to reduce the long list to a much smaller and more tractable list of key factors; and second, a $2 \mathrm{VII}^{6-1}$ fractional factorial design, carried out to test the effects of only the 6 more relevant key factors identified previously.

After said comprehensive preliminary screening based on a "one-factor-at-a-time" approach, six variables were further tested using a $2{ }_{\mathrm{VI}}{ }^{6-1}$ fractional factorial design; yeast extract, peptone, temperature and $\mathrm{pH}$ were eventually found to affect protease production to a significant degree. This experimental approach proved adequate in statistical terms - and a significant improvement (viz. 2.2-fold) quite comparable to the most successful studies on similar topics, could be accomplished in the level of 
protease production. Further detailed discussion of the procedure followed and the results generated is provided below.

\subsection{Significant Physicochemical Parameters}

None of the several carbon sources tested yielded as good a protease productivity as the NB medium formulation - thus indicating that presence of other constituents in the medium instead of beef extract either inhibit protease production, or at least fail to promote it. Note that protease synthesis was reduced by ca. $93 \%$ in the presence of glucose, probably due to catabolite repression [15] - even though Son et al. (2008) have reported otherwise.

Since the results encompassing protease production with yeast extract and peptone together had suggested synergistic action, both nutrients were selected for the subsequent optimization step. On the other hand, $\mathrm{pH} 7$ seemed to be optimum, but as a significant factor upon enzyme production, it was also considered for the $2 \mathrm{VI}^{6-1}$ fractional factorial design afterwards.

It is known that stirring promotes oxygen solubility, disruption of air bubbles putatively formed and dispersion of cell clusters [16] — which may favourably contribute to protease synthesis, by facilitating access of cells to the nutrients in the medium while avoiding inhibitory product concentration build-up. However, high stirring rates are also known to cause cell disruption, and this difficulty may overrun the abovementioned advantages of stirring. The essentially similar results obtained for protease production under high stirring rates and no stirring at all may instead be due to little oxygen availability and intrinsic difficulty in accessing nutrients. Since agitation is an important factor toward economically feasible industrial processes, stirring rate was picked as a factor to proceed with.

While $37^{\circ} \mathrm{C}$ appeared to be optimum, it became clear that temperature is a significant factor upon protease production, so it was included in the $2_{\mathrm{VI}}^{6-1}$ fractional factorial design.

Inoculum density seemed to have some impact upon the enzyme production level, and it is surely an important parameter to be considered for industrial scale up; hence, this factor was also included in subsequent optimization studies.

After the "one-factor-at-a-time" approach to the effect of individual NB constituents, it was quite difficult to draw a conclusion on which nutrients should be selected for the next optimization step. Therefore, a further assessment of their interaction was carried out, via a fractional factorial design with four independent factors only. The decision on the concentration ranges (i.e. the lower, -1 , and the upper, +1 , levels) to be considered in this experiment was based on the previous results of screening experiments with NB medium as central point; in view of our purpose here, each was performed only once.

The response to beef extract $\left(X_{1}\right)$ and yeast extract $\left(X_{2}\right)$ levels, in terms of protease production $(Y)$ by Bacillus sp. HTS 102, was according to:

$$
Y=-9.26+38.62 X_{1}+7.76 X_{2}-7.33 X_{1} X_{2}
$$

This regression equation held a good fit, as its multiple correlation coefficient $\left(R^{2}\right)$ is 0.9695 ; recall that a value above 0.75 indicates an acceptable fit of the model to the experimental data [8]. This coefficient is in fact an estimate of the fraction of overall variation in the data that can be accounted for by the model, so our model was able to statistically explain $96.95 \%$ of the variation in the responses. The value of 0.9466 for the "adjusted $\mathrm{R}^{2 \text { ", }}$ further confirms significance of the above model, which

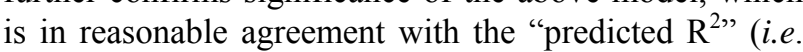
0.8780 ). The "adequate precision value" of our model was 12.46 , thus suggesting that it can be safely used to "navigate" through the design space [8]. The negative coefficient associated with the two-factor interaction term $\left(X_{1} X_{2}\right)$ is also consistent with the results displayed in Figure 4.

Since there was no statistically significant difference between effects at the upper limit $(+1)$ of any of the two variables, beef extract was excluded from the next step of optimization for economic reasons - as it is the more expensive nutrient. Consequently, further optimization studies resorted only to a single concentration of beef extract $\left(0.5 \mathrm{~g} \cdot \mathrm{L}^{-1}\right)$. Furthermore, the effects of $\mathrm{NaCl}$ and peptone upon protease activity were found below the $t$-value threshold; however, the latter concentration was selected anyway as factor for the next optimization step, since it seemed important to further investigate its role upon enzyme production. On the other hand, $\mathrm{NaCl}$ was fixed at its lowest level (i.e. $1.0 \mathrm{~g} \cdot \mathrm{L}^{-1}$ ), because high levels can induce osmotic unbalance in bacteria and thus raise the cost associated with medium formulation.

In view of the considerations above, the combined effects of yeast extract, peptone, inoculum, stirring, temperature and $\mathrm{pH}$ were picked up as key determinants for protease production, and were accordingly included in the next fractional factorial design.

\subsection{Optimization via $2 \mathrm{vi}^{6-1}$ Fractional Factorial Design}

Following normalization of the data by a square root transformation, the ANOVA results shown in Figure 6 allowed one to draw four major conclusions: 1) variations in the levels of inoculum or stirring rate did not significantly affect protease production, so they were removed from the final model; 2) protease production was significantly affected by yeast extract, peptone, 
temperature and $\mathrm{pH}$, at a significance level of $0.1 \% ; 3$ ) the effect of temperature was positive, thus implying that its increase will promote protease production-whereas the effect of the linear model term for peptone was negative, so protease production will be enhanced by lower levels thereof; and 4) interaction between yeast extract and $\mathrm{pH}$ produced a (negative) effect upon protease production that was stronger than either of their linear effects (Figure 7(a)) - which is consistent with the claim [17] that extracellular secretion of proteases in Bacillus spp. is a manifestation of nitrogen limitation prevailing at the onset of the stationary phase. (Note that only the effect of yeast concentration and $\mathrm{pH}$ were considered in this figure-for a given set of values of all other variables).

An interaction between factors occurs when the overall response is different from their combination consid-

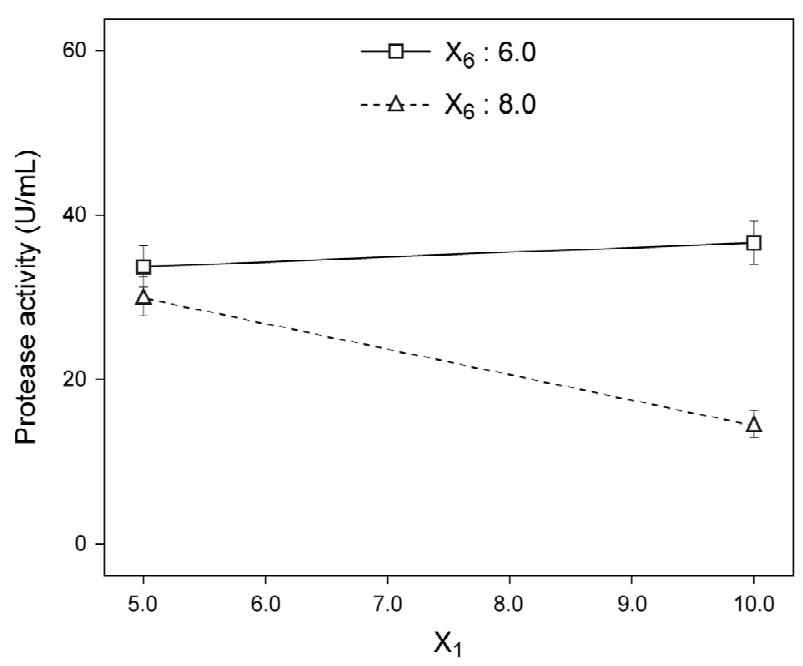

(a)

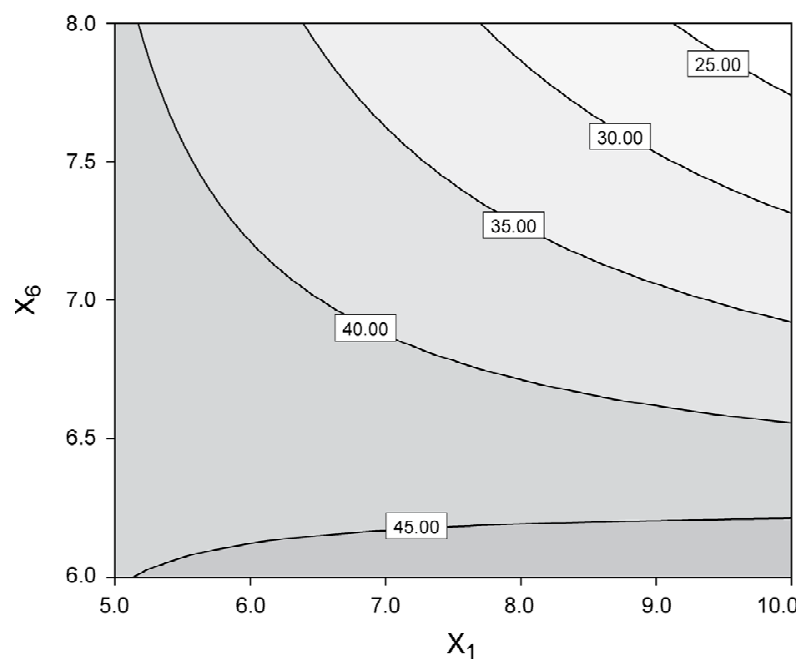

(b)

Figure 7. Best fit model plots: (a) Interaction plot; and (b) Contour plot associated with the significant $\mathrm{X}_{1} \mathrm{X}_{6}$ interaction. ered independently. Interaction plots, as is the case of Figure 7(a), make it easy to interpret two-factor interactions, as they exhibit non-parallel lines whenever the effect of one factor depends on the level of the other; hence, the interaction between yeast extract and $\mathrm{pH}$ proved significant. The contour plot represented in Figure 7(b) is a two-dimensional representation of the response across the selected factors; apparently the surface is not symmetrical, and no peak can be perceived. Therefore, room apparently exists to further fine tune the optimal loci predicted from our final model found using RSM.

\section{ACKNOWLEDGEMENTS}

Author A. C. Queiroga acknowledges a PhD fellowship (ref. SFRH/ BD/19121/2004), granted by Fundação para a Ciência e a Tecnologia (Portugal) and supervised by author F. X. Malcata.

\section{REFERENCES}

[1] Ibrahim, A.S.S. and Al-Salamah, A.A. (2009) Optimization of media and cultivation conditions for alkaline protease production by alkaliphilic Bacillus halodurans. Research Journal of Microbiology, 4, 251-259. doi:10.3923/jm.2009.251.259

[2] Gupta, R., Beg, Q. and Lorenz, P. (2002) Bacterial alkaline proteases: Molecular approaches and industrial applications. Applied Microbiology and Biotechnology, 59, 15-32. doi:10.1007/s00253-002-0975-y

[3] Navaneeth, S., et al. (2009) Optimization of medium for the production of subtilisin from Bacillus subtilis MTCC 441. African Journal of Biotechnology, 8, 6327-6331.

[4] Shafee, N., et al. (2005) Optimization of environmental and nutritional conditions for the production of alkaline protease by a newly isolated bacterium Bacillus cereus strain 146. Journal of Applied Sciences Research, 1, 1-8.

[5] Huang, G.R., et al. (2008) Optimization of medium composition for thermostable protease production by Bacillus sp. HS08 with a statistical method. African Journal of Biotechnology, 7, 1115-1122.

[6] Queiroga, A.C., Pintado, M.E. and Malcata, F.X. (2012) Potential use of wool-associated Bacillus species for biodegradation of keratinous materials. International Biodeterioration and Biodegradation, 70, 60-65. doi:10.1016/j.ibiod.2011.12.013

[7] Joo, H.-S. and Chang, C.-S. (2005) Production of protease from a new alkalophilic Bacillus sp. I-312 grown on soybean meal: Optimization and some properties. Process Biochemistry, 40, 1263-1270. doi:10.1016/j.procbio.2004.05.010

[8] Reddy, L.V.A., et al. (2008) Optimization of alkaline protease production by batch culture of Bacillus sp. RKY3 through Plackett-Burman and response surface methodological approaches. Bioresource Technology, 99, 22422249. doi:10.1016/j.biortech.2007.05.006

[9] Rao, Y.K., et al. (2007) Medium optimization of carbon 
and nitrogen sources for the production of spores from Bacillus amyloliquefaciens B128 using response surface methodology. Process Biochemistry, 42, 535-541. doi:10.1016/j.procbio.2006.10.007

[10] Puri, S., Beg, Q.K. and Gupta, R. (2002) Optimization of alkaline protease production from Bacillus sp. by response surface methodology. Current Microbiology, 44, 286-290. doi:10.1007/s00284-001-0006-8

[11] Myers, R.H. and Montgomery, D.C. (2002) Response surface methodology: Process and product optimization using designed experiments. Wiley Series in Probability and Statistics, Wiley Interscience, New York, 824.

[12] Queiroga, A.C., Pintado, M.M. and Malcata, F.X. (2007) Novel microbial-mediated modifications of wool. Enzyme and Microbial Technology, 40, 1491-1495. doi:10.1016/j.enzmictec.2006.10.037

[13] $\mathrm{Gu}, \mathrm{X} . \mathrm{B}$., et al. (2005) Optimization of medium constituents for a novel lipopeptide production by Bacillus subtilis MO-01 by a response surface method. Process Biochemistry, 40, 3196-3201. doi:10.1016/j.procbio.2005.02.011

[14] Kumar, C.G. and Takagi, H. (1999) Microbial alkaline proteases: From a bioindustrial viewpoint. Biotechnology Advances, 17, 561-594. doi:10.1016/S0734-9750(99)00027-0

[15] Chi, Z., et al. (2007) Optimization of medium and cultivation conditions for alkaline protease production by the marine yeast Aureobasidium pullulans. Bioresource Technology, 98, 534-538. doi:10.1016/j.biortech.2006.02.006

[16] Joo, H.-S. and Chang, C.-S. (2006) Production of an oxidant and SDS-stable alkaline protease from an alkaophilic Bacillus clausii I-52 by submerged fermentation: Feasibility as a laundry detergent additive. Enzyme and Microbial Technology, 38, 176-183. doi:10.1016/j.enzmictec.2005.05.008

[17] Liu, J., et al. (2005) Optimization of nutritional conditions for nattokinase production by Bacillus natto NLSSE using statistical experimental methods. Process Biochemistry, 40, 2757-2762. doi:10.1016/j.procbio.2004.12.025 